\title{
Efficient Relay Selection Scheme based on Fuzzy Logic for Cooperative Communication
}

\author{
Shakeel Ahmad Waqas \\ Military College of Signals \\ National University of Sciences and Technology (NUST) \\ Rawalpindi/Islamabad, Pakistan
}

\author{
Imran Touqir \\ Military College of Signals \\ National University of Sciences and Technology (NUST) \\ Rawalpindi/Islamabad, Pakistan
}

\author{
Nasir Khan \\ Department of telecommunication Engineering \\ University of Engineering and Technology (UET) \\ Mardan/Peshawar, Pakistan
}

\author{
Imran Rashid \\ Military College of Signals \\ National University of Sciences and Technology (NUST) \\ Rawalpindi/Islamabad, Pakistan
}

\begin{abstract}
The performance of cooperative network can be increased by using relay selection technique. Therefore, interest in relay selection is sloping upward. We proposed two new relay selection schemes based on fuzzy logic for dual hop cooperative communication. These relay selection schemes require SNR (signal to noise ratio), cooperative gain and channel gain as input fuzzy parameters for selection of best relay. The performance of first proposed relay selection scheme is evaluated in term of BER (bit error rate) in Nakagami, Rician and Rayleigh fading channels. In second proposed relay selection scheme, threshold is used with the objective to minimize the power consumption and channel estimation load. Its performance is analyzed in term of BER, number of active relays and load of number of channel estimations.
\end{abstract}

Keywords-Cooperative Networks; Relay selection schemes; Amplify and forward; Fuzzy logic; Nakagami Fading channel; Rician Fading Channel; Rayleigh Fading Channel

\section{INTRODUCTION}

In future wireless communication networks, multipath fading is the key problem to achieve high data rate. Time, frequency and spatial diversity techniques are considered to alleviate the multipath fading. Taking specifically spatial diversity, which normally improves the system by introducing independent path communication. Primitively, the concept was achieved by introducing multiple antennas (MIMO) which improves the performance manifolds. Due to size, cost along with some hardware limitations, the system practical implementation becomes another problem itself. For overcoming these concerns, cooperative communication was introduced as a virtual MIMO environment [1]. For bringing independent path communication into being for practical implementation and exploiting the broadcast nature of the wireless communication destination relays are introduced between source and destination, which in literal sense are not more than forwarders of the source signal based on some designed protocol [2].

Relaying protocols are followed by the relay for forwarding of the signal to destination which includes namely "Amplify and Forward" (AF), "Decode and Forward" (DF), "Estimate and Forward", "Compressed and Forward" etc. [3]. For minimizing complexity of the system, AF is the best technique which forward the amplified received signal of the source to destination with the demerit of noise also being get amplified along with the signal [4] [2] [5] [6] [7, 8]. For better performance DF is normally taken into account which decodes the signal at the relay and then encode it back before forwarding [2, 4] [9] [10] [11]. In practical sense, more than one relays are present and use of all relays will leads to interference with the sources, high power consumption and consuming high bandwidth. For covering up this problem a relay with better specifications and according to the requirements of the application is chosen which forwards the signal from source to destination.

Relay selection is studied extensively these days by researchers and magnificent work can be found in [12-28] .After analysis the literature, we can in general classify the work done on relay selection into five categories based on the kind of selection technique used:

\section{a) Geographical information based relay selection}

Geographical information based relay selection with the aim to minimize symbol error probability was presented by Wang et al. which used the distance from source to relay and from rely to destination as a criterion for best relay selection. But because of channel fading and shadowing effect the proposed algorithm is not applicable for practical scenario[12]. Three-time slot TDMA based transmission protocol has been investigated and analyzed by U. R. Tanoli et al. with the better performance than old protocols using the location information. The deficiency of the protocol is an extra time slot for transmission thus decreasing the code rate and also impractical because of location based information [13]. Analysis of $\mathrm{AF}$ and DF relaying protocols for inter-relay communication with the proof that AF can perform better than DF in term of BER is presented in [14].

\section{b) Energy efficiency based relay selection}

The expression of total energy is obtained and used for relay selection in general scenario with characterization of the structure for optimal transmission [15]. Power aware relay 
selection is proposed by Yan Chen et al. with the aim of minimizing the overall network life-time. Furthermore, optimal power allocation and three power-aware selection criteria are being analyzed [16]. Relay selection on the basis of channel state information with the contribution of power allocation algorithm, for the purpose of presenting an energy-saving relay selection strategy. The solution presented is well analyzed but location based information is also used. However computational complexity and requirement of information at the source is not fully addressed [17].

\section{c) Outage probability based relay selection}

Optimal-outage relay selection scheme is presented by $\mathrm{Li}$ Sun et al. with utilization of feedback from the receiver for the decision of whether rely cooperation is required or not. Adaptive $\mathrm{DF}$ and $\mathrm{AF}$ is used in this paper as forwarding scheme [18]. However, outage priority based fairness is proposed by $\mathrm{Li}$ Yubu et al. with the aim to improve relay selection fairly without performance disturbance and with improvement in network lifetime [19]. The effect of correlated log-normal shadowing is analyzed based on outage probability using opportunistic DF and showing significant impact on outage performance [20].

\section{d) Interference aware relay selection}

Interference is pretty common to exist in wireless communication and have an unavoidable effect over the performance of the communication in case of multiple transmission pairs. Relay Interference effect in cooperative networks is analyzed by $\mathrm{Y}$. Zhu and $\mathrm{H}$. Zheng between interference management and cooperative relay strategy and two spectrum selection techniques are presented with different tradeoffs. However, the paper does not deal the problem of relay selection at all [21]. Furthermore, interference based relay selection is devised with the purpose of maximizing the mutual information on cooperative networks with the interference limited destination [22]. Interference aware relay selection is proposed with the use of distributed interference aware relay selection algorithm by $\mathrm{C}$ Shi et al in [23] to select best relay by using inter node interference and channel statistics.

\section{e) Channel state information based relay selection}

Opportunistic relaying was presented in [24] as the best scheme for relay selection in which the source have exact information of source to relay and then relay to destination, only then source was able to select the best relay out of all the relays. The system delay during selection and not exact surety of same channel during data transmission as was during estimation were the major issues not addressed in the paper. The authors in [25] have utilized the outdated channel state information for selection of best relay and have adopted maximum a posteriori for the prediction of actual SNR during the transmission and have utilized it for single relay selection as a strategy. An algorithm is proposed in [26] in which instead of using global knowledge of all the paths local channel states available at the relay is utilized for relay to select and mark itself as the best relay thus implementing distributed relay selection. SNR is calculated using outdated method of SNR calculation and then according to length of data transmission and channel state information available best relay is selected thus reducing the repeated relay selection technique [27].
Furthermore, Glauber Brante et. al. reported a fuzzy logic based relay selection algorithm for wireless cooperative sensor network. CSI (channel state information) and residual energy are the input fuzzy parameter of fuzzy controller [28].

To the best of our knowledge, the discussed relay selection techniques are causing enormous control message overhead because of repeatedly relay selection for cooperation, when successive data transmission is carried out especially in case of audio or video session occurring between source and destination, thus causing degradation in network performance. To reduce the frequent selection of relays we propose two algorithms based on local channel state (CSI) information available with the use of fuzzy logic algorithm for computational complexity and control message overhead reduction while promising for selection of best relay and guaranteeing betterment in symbol error rate. Three fuzzy parameters are used in the defined algorithms namely signal to noise ratio (SNR), cooperative gain and channel gain. Cooperative gain is defined here as the ratio of direct transmission bit error rate (BER) to cooperative transmission BER. The performance of proposed relay selection scheme is analyzed and compared on dual hop cooperative network over three fading channels namely Rayleigh, Rician and Nakagami. For reduction of control message overhead and power consumption, another modified algorithm is proposed with the use of threshold. Moreover, number of active relays and number of channel estimation depend on threshold chosen. The tradeoff curves of threshold, BER, number of active relays and channel estimation are presented in this paper. AF is used as relaying protocol and MRC (maximal ratio combining) is selected as combining technique at destination. The performance of both relay selection schemes are evaluated through Monte Carlo simulation. Comparison with previous relay selection schemes is also carried out in this research.

Rest of the paper is organized in the following pattern. Section 2 defines the system model proposed in this research. Whereas relay selection criterion and selection algorithms are discussed in section 3. Section 4 comprises of the simulation model and simulation parameters while simulation results are discussed in section 5. Paper is concluded and future work is presented in section 6 .

\section{SYSTEM MODEL}

Considering the cooperative communication, our proposed system model investigated in this paper consist of a source (S) transmitting its signal to destination (D) through the cooperation of $\mathrm{N}$ relays $R_{i}$ wheras $(i=1,2,3 \ldots N)$ as shown in Fig. 1. with the consideration that each terminal is mounted with single antenna. An assumption is taken that all the relays are operating in half duplex mode and let $h_{s-d}, h_{s-r i}$ and $h_{r i-d}$ are the channel coefficients from source to destination, source to $i^{\text {th }}$ relay and form $i^{\text {th }}$ relay to destination respectively. It is also assumed that zero mean white Gaussian noise is there at each terminal. As The network model is dual hop with the consideration that no inter relay communication is occurring and the source knows the channel statistics of both the relays using the loop feedback. Considering $E_{S}$ as transmission power of the source while $E_{r_{i}}$ as the transmission power of the $i^{\text {th }}$ relay. Now let $X$ be the signal transmitted by source and $Y_{D}$ is 
the signal received at destination then as discussed in [2] the signal received at destination can be represented as:

$$
Y_{D}=h_{s-d} * \sqrt{E_{s}} X+n_{d}
$$

Whereas $n_{d}$ is the noise at destination. Now the signal received at $i^{\text {th }}$ relay will be:

$$
Y_{r_{i}}=h_{s-r_{i}} * \sqrt{E_{s}} X+n_{r_{i}}
$$

Now as according to the considered relaying protocol AF, an amplified copy of the received signal amplified with a factor of $\beta$ at $i^{\text {th }}$ relay will be forwarded towards destination which can be represented as:

$$
\begin{gathered}
Y_{D-r_{i}}=h_{r_{i}-D} * \sqrt{E_{r_{i}}} \beta\left(h_{s-r_{i}} * \sqrt{E_{s}} X\right)+h_{r_{i}-D} * \\
\sqrt{E_{r_{i}}} \beta n_{r_{i}}+n_{D}
\end{gathered}
$$

Two time slots model is considered for avoidance of interference in which signal is broadcasted by the source at time slot 1 and received by both the destination and the $i^{\text {th }}$ relay. While in $2^{\text {nd }}$ time slot amplified copy of the received signal is transmitted by the relay. Multiple copies of source signal are received at destination and are combined together using MRC technique while ignoring any time delay occurred during transmission. For simplicity, we assume the path loss component equal to 1 . Using proposed relay selection algorithm, best relay out of all will be selected for cooperation by the destination and the information will be sent to all the relays along with source using feedback transmission.

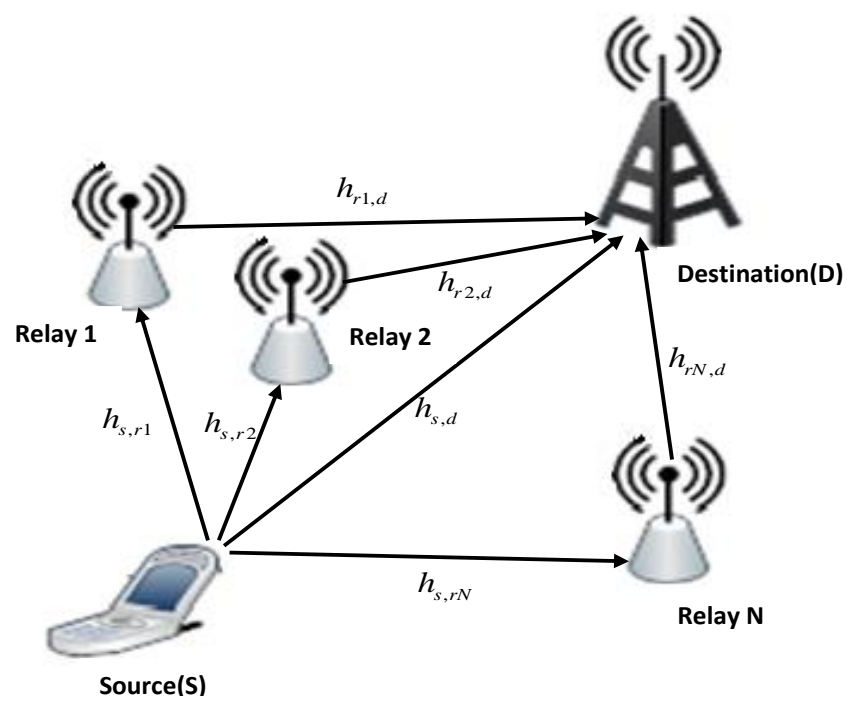

Fig. 1. System Model

\section{RELAY SELECTION CRITERION AND AlgORITHMS}

A cooperative communication model was discussed in the previous section. Now the scenario is that we will be having $N$ number of relays. Out of all the available relay one relay which can outperform other on the basis of performance will be selected for cooperation by using the algorithms discussed here. But before discussing relay selection algorithms first we will discuss the following parameters which are used for relay selection on the basis of the defined algorithms:

\section{B. SNR}

The SNR of direct path i.e. transmission of signal from source to destination is denoted by $\gamma_{S-D}$ and can be written as[29]:

$$
\gamma_{S-D}=\frac{\left|h_{S-D}\right|^{2}}{N_{D}}
$$

The SNR of 1 st hop of $i^{\text {th }}$ relay is denoted by $\gamma_{S-r_{i}}$ and its equation is:

$$
\gamma_{S-r_{i}}=\frac{\left|h_{S-r_{i}}\right|^{2}}{N_{r_{i}}}
$$

The amplified signal is forwarded to destination in 2nd hop. The SNR of 2 nd hop of $i^{t h}$ relay is represented by $\gamma_{r_{i}-D}$ and is given as:

$$
\gamma_{r_{i}-d}=\frac{\beta\left|h_{S-r_{i}}\right|^{2}\left|h_{r_{i}-D}\right|^{2}}{\left(\beta^{2}\left|h_{S-r_{i}}\right|^{2}+1\right) N_{r_{i}}}
$$

For the relay selection, the minimum SNR of two hop is used. The SNR value of $i^{\text {th }}$ relay used for relay selection is given below:

$$
\gamma_{i}=\min \left(\gamma_{S-r_{i}}, \gamma_{r_{i}-d}\right)
$$

\section{Cooperative Gain}

Cooperative gain is defined as the ratio of BER of direct transmission to BER of cooperative transmission. The BER of $i^{\text {th }}$ relay used for relay selection can be written as:

$$
C G_{i}=\frac{B E R_{\text {direct }}}{B E R_{\text {Cooperative }}}
$$

\section{Channel Gain}

For the proposed relay selection scheme, the minimum channel gain of two hop is considered. For $i^{\text {th }}$ relay, the channel gain used for relay selection is:

$$
h_{i}=\min \left(h_{S-r_{i}}, h_{r_{i}-d}\right)
$$

The relay selection scheme proposed in this work is making use of fuzzy logic, which consists of input fuzzy parameters, fuzzification, fuzzy inference system, fuzzy rules and defuzzification. SNR, cooperative gain and channel gain are the three input fuzzy parameters. In the process of fuzzification, a static value is assigned to variables $\gamma_{i}, C G_{i}$ and $h_{i}$ defined by the input membership functions. SNR is having five membership functions (V.Low, Low, Medium, High, V.High), cooperative gain is having three membership functions (Poor, Normal,Good), while channel gain is also having three input membership functions (Bad,Moderate, Best) and by calculation total 45 rules are defined for it. The degree of relevance $f\left(\gamma_{i}, C G_{i}, h_{i}\right)$ is calculated considering the strength of each rule and the output membership functions (Not Selected, Considered, Selected) which are shown in Fig. 3. The higher $f\left(\gamma_{i}, C G_{i}, h_{i}\right)$, higher will be the "quality" of the selected relay. Both the algorithms are shown in Fig. 2. Along with block diagram of relay selection scheme.

Algorithm I:

Step 1: Initiate $i=0$

Step 2: Increment $i$ by 1 . 
Step 3: Calculate the fuzzy parameters for $i^{\text {th }}$ relay.

Step 4: Find out the degree of relevance

Step 5: If $i=N$, then jump to step 6, else jump to step 2 .

Step 6: Compare the degree of relevance of each relay and select the relay having highest degree of relevance.

End

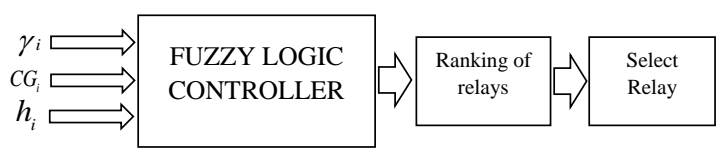

(a)

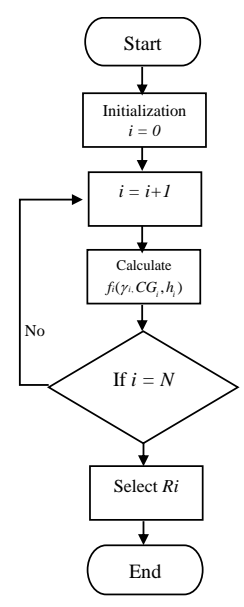

(b)

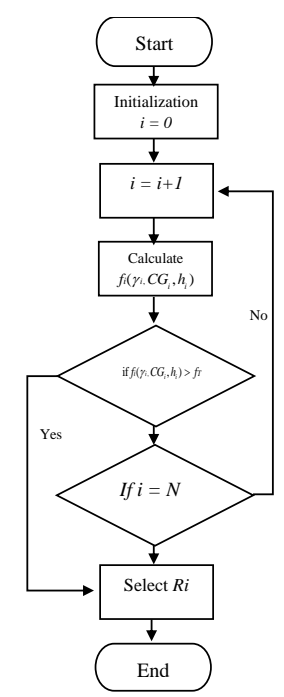

(c)

Fig. 2. (a) Block diagram of relay selection scheme (b) Algorithm-1(c) Algorithm-2

In this algorithm, $2 \mathrm{~N}$ channels estimation must be conducted for selecting best relay and all the relays need to be in $\mathrm{ON}$ state. In order to reduce the load of channel estimation and power consumption, another relay selection algorithm is presented, in which use of predetermined threshold $f_{r}$ is carried out. This scheme has four advantages over the previous. Channel estimation load i.e. number of relays whose path coefficients are taken into account, is reduced, no need to turn $\mathrm{ON}$ all the relays, time for relay selection is reduced and calculations for all the relays is reduced. Steps of this algorithm are given below:

Algorithm II:

Step 1: Initiate $i=0$

Step 2: Increment $i$ by 1

Step 3: Calculate the fuzzy parameters for $i^{\text {th }}$ relay

Step 4: Find out the degree of relevance $f\left(\gamma_{i}, C G_{i}, h_{i}\right)$

Step 5: If $f\left(\gamma_{i}, C G_{i}, h_{i}\right)>f_{r}$, stop the calculation and go to step 8, else continue

Step 6: Else-if $i=N$, then continue, otherwise jump to step 2

Step 7: Compare the degree of relevance of each relay and select the relay having highest degree of relevance
Step 8: $R_{i}$ is selected as best relay

End

Mamdani fuzzy inference system (FIS) [30] is used in both the algorithms for fuzzification. All the defined rules have equal weightage in calculating the degree of relevance and for defuzzification centroid approach is used.

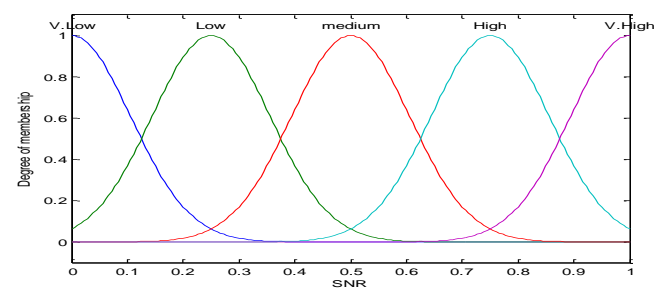

(a)

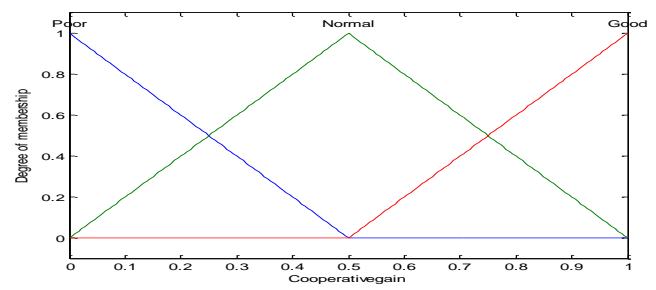

(b)

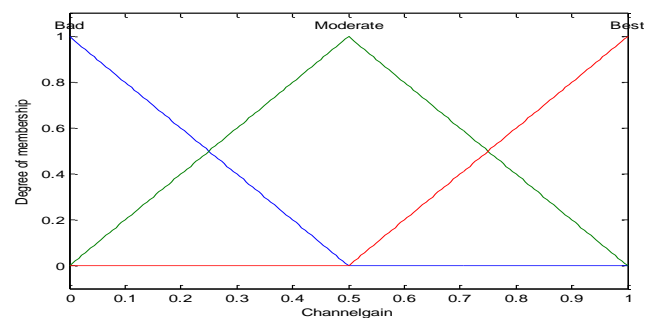

(c)

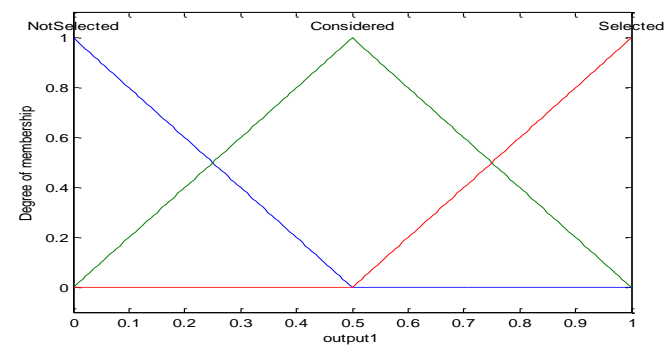

(d)

Fig. 3. Membership function (a) SNR (b) CGi (c) hi (d) degree of relevance

\section{SimUlation MODEL AND PARAMETERS}

In simulation model as shown in Fig. 4. we can observe that total of 5 relays are considered in-between source and destination. The model is showing the link from source to destination, source to relay and relay to destination. Each link is shown with its channel coefficient and the additive white Gaussian noise is also shown. Monte Carlo simulation are carried out for both the algorithms using the same simulation model. Amplify and forward as relaying protocol while B-PSK as a modulation scheme. During simulation transmission power was kept constant of 1watt for every node while the 
amplification factor is kept fixed. Additive white Gaussian noise is used at each node and maximal ratio combining is used as receiver diversity whereas $10^{\wedge} 6$ bits are transmitted from source to destination for every simulation carried out. Both the algorithms are analyzed for three channel models namely Rayleigh, Nakagami and Rician. For Nakagami fading channel $\mathrm{m}$ is assigned value of 3 while $\mathrm{k}$ was assigned value of 5 for Rician fading channel. All these parameters are summarized in Tab. 1 .

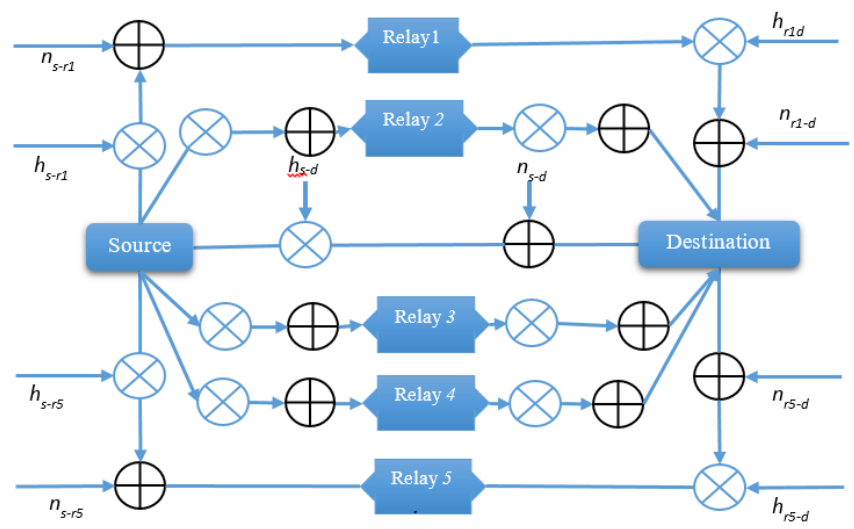

Fig. 4. Simulation Model

TABLE I. SIMULATION PARAMETERS

\begin{tabular}{|l|l|}
\hline Parameters & Values \\
\hline Source Transmit Power & $1 \mathrm{w}$ \\
\hline Relay Transmit Power & $1 \mathrm{w}$ \\
\hline Amplification Factor & Fixed \\
\hline Modulation Scheme & B-PSK \\
\hline Receiver Diversity & MRC \\
\hline Number of Relays & 5 \\
\hline Relaying Protocol & Amplify-and-Forward \\
\hline Number of Bits & $10^{\wedge} 6$ \\
\hline Receiver Noise & AWGN \\
\hline Channel Model & $\begin{array}{l}\text { Rayleigh, Nakagami, } \\
\text { Rician }\end{array}$ \\
\hline
\end{tabular}

\section{SimUlation RESUltS AND DISCUSSION}

Fig. 5.(a) shows the SER analysis of algorithm-1 over rayleigh, rician and nakagami fading channels. At low SNR values, better performance is observed in nakagami fading channel and at high SNR values, better performance is observed in rician fading channel. The reason behind this is the fact that is the line of sight component always have better performance at higher SNR regions. While Rayleigh which is more practical model, is having comparable results when cooperation is taken into account and compared with direct path of Rician. The SER analysis of algorithm-II, considering the threshold $\left(f_{r}\right)$ equal to 0.7 is shown in Fig. 5.(b) is following exactly the same fashion as was observed in algorithm-1, reason for which is no change in channel models only reduction of computations and time of relay selection. The proposed relay selection, selected that relay which showed low
SER in Nakagami fading channel at low SNR and at high SNR, low SER is observed in Rician fading channel. While along with cooperation, Rayleigh is again following the footprints of direct path communication of Rician fading channel.

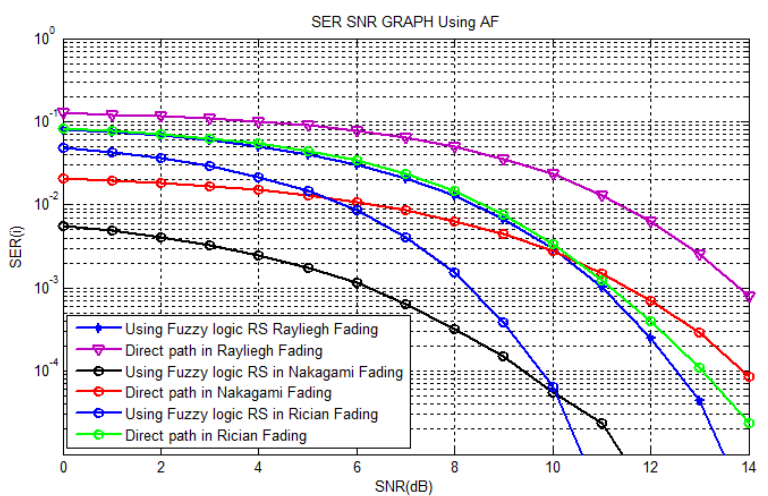

(a)

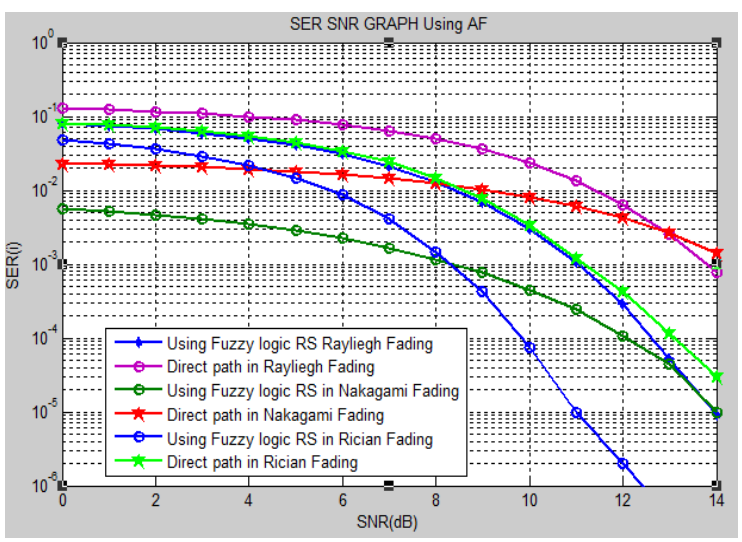

(b)

Fig. 5. SER analysis using Rayleigh, Rician and Nakagami fading channels using $f r=0.7$ (a) Algorithm-I (b) Algorithm-II

Fig. 6. shows the relation of threshold $\left(f_{r}\right)$ with number of channel estimations, number of active relays and SER. This relationship is evaluated in Rayleigh fading channel using the SNR value equal to 1 and is only applicable for algorithm-2. Our simulation result proved that number of channel estimations and number of active relays (power consumption) increases as the threshold $\left(f_{r}\right)$ increases. Fig. 6.(a) shows increase in the average number of active relays as threshold $\left(f_{r}\right)$ increases reason for which is the fact that with increase in threshold, the algorithm will evaluate more and more relays to find the fittest one. Fig. 6.(b) shows the average number of channels estimated for relay selection and threshold $\left(f_{r}\right)$ curve with the same trends and reasons as was for Fig. 6.(a) discussed above. Fig. 6.(c) shows a decrease in the average SER as the threshold $\left(f_{r}\right)$ increases as the fittest on the relays will be selected with the increase in threshold. 


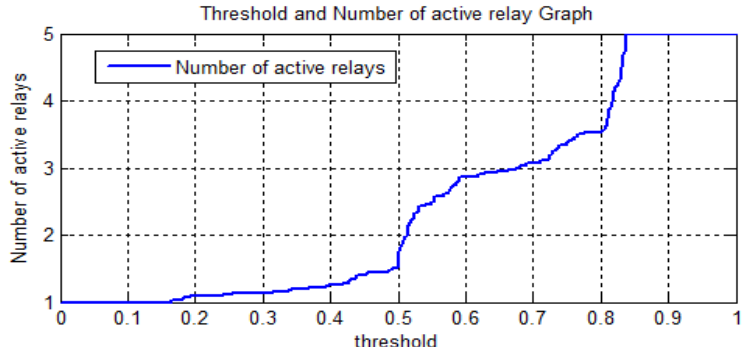

(a)

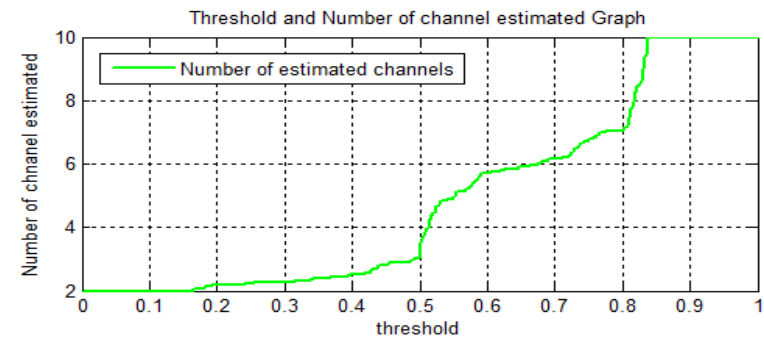

(b)

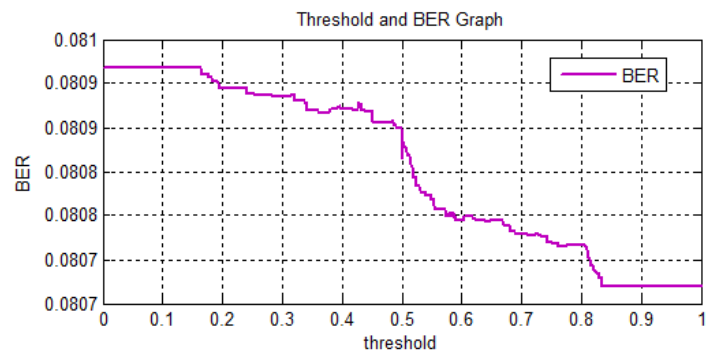

(c)

Fig. 6. In Raleigh fadding enviroment curve of threshold vs (a) Activerelays (b) number of channel estimations (c) BER

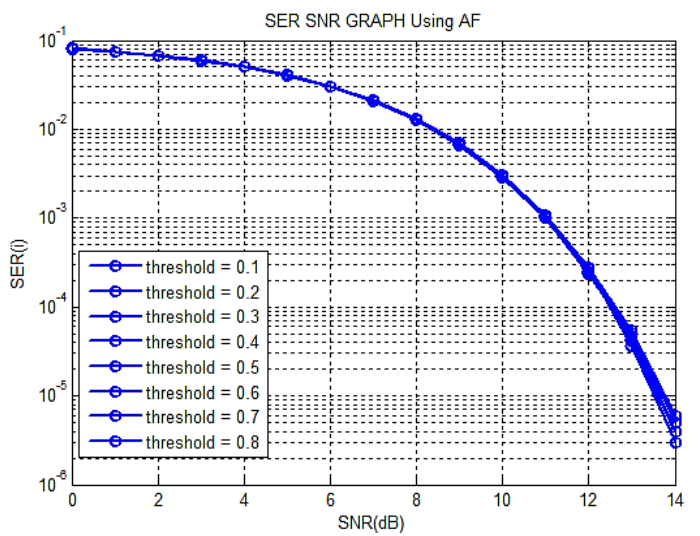

Fig. 7. BER analysis of algorithm-II in Rayleigh fading channel using different value of threshold

As clear from the figures that if we want low power consumption, less channel estimation (low load, less processing time for relay selection), system performance will become low in term of SER. To analyze the degradation of system performance in term of BER, performance using Rayleigh fading channel for different values of threshold $\left(f_{r}\right)$ is evaluated. It is proved through our simulation result that the
SER curves for different values of threshold $\left(f_{r}\right)$ have very minute difference and almost equal at low SNR values. Fig. 7. shows the BER curve for different values of threshold $\left(f_{r}\right)$.

\section{CONCLUSION AND FUTURE WORK}

In this paper, fuzzy logic based relay selection schemes are presented using three input fuzzy parameters i.e. SNR, cooperative gain and channel gain. In second proposed algorithm, threshold is used in order to minimize the power consumption and channel estimation required for relay selection. Simulation result showed that number of active relays and load of channel estimation is decreased by using the threshold. And also number of active relays are reduced so conserving power. SER is poorly effected at high SNR and almost not effected at low SNR. In future, this work can be extended by including the secrecy constraints in proposed relay selection schemes. Whereas, multi-hop communication in this system with multi-user detection and supplementary cooperation could increase the performance of the system manifolds while also increase in code rate is expected.

\section{REFERENCES}

[1] Politis, C., et al., Cooperative networks for the future wireless world. IEEE Communications Magazine, 2004. 42(9): p. 70-79.

[2] Laneman, J.N., D.N. Tse, and G.W. Wornell, Cooperative diversity in wireless networks: Efficient protocols and outage behavior. IEEE Transactions on Information theory, 2004. 50(12): p. 3062-3080.

[3] Kharat, P. and J. Gavade, Cooperative communication: New trend in wireless communication. International Journal of Future Generation Communication and Networking, 2013. 6(5): p. 157-166.

[4] Laneman, J.N. and G.W. Wornell, Distributed space-time-coded protocols for exploiting cooperative diversity in wireless networks. IEEE Transactions on Information theory, 2003. 49(10): p. 2415-2425.

[5] Hasna, M.O. and M.-S. Alouini, End-to-end performance of transmission systems with relays over Rayleigh-fading channels. IEEE Transactions on Wireless Communications, 2003. 2(6): p. 1126-1131.

[6] Zhao, Y., R. Adve, and T.J. Lim. Improving amplify-and-forward relay networks: optimal power allocation versus selection. in 2006 IEEE International Symposium on Information Theory. 2006. IEEE.

[7] Zhao, Y., R. Adve, and T.J. Lim, Symbol error rate of selection amplifyand-forward relay systems. IEEE Communications Letters, 2006. 10(11): p. 757-759.

[8] Krikidis, I., et al., Amplify-and-forward with partial relay selection. IEEE Communications Letters, 2008. 12(4): p. 235-237.

[9] Duong, T.Q., V.N.Q. Bao, and H.-j. Zepernick, On the performance of selection decode-and-forward relay networks over Nakagami-m fading channels. IEEE Communications Letters, 2009. 13(3): p. 172-174.

[10] Su, W., A.K. Sadek, and K.R. Liu. SER performance analysis and optimum power allocation for decode-and-forward cooperation protocol in wireless networks. in IEEE Wireless Communications and Networking Conference, 2005. 2005. IEEE.

[11] Luo, J., et al., Decode-and-forward cooperative diversity with power allocation in wireless networks. IEEE transactions on wireless communications, 2007. 6(3): p. 793-799.

[12] Wang, C.-L. and S.-J. Syue. A geographic-based approach to relay selection for wireless ad hoc relay networks. in Vehicular Technology Conference, 2009. VTC Spring 2009. IEEE 69th. 2009. IEEE.

[13] Tanoli, U., et al., Performance analysis of cooperative networks with inter-relay communication over Nakagami-m and rician fading channels. International Journal on Multidisciplinary sciences, 2012. 3(4): p. 24-29.

[14] Tanoli, U., et al., Comparative analysis of fixed-gain relaying schemes for inter-relay communication over Nakagami-m fading channel. Sindh University Research Journal-SURJ (Science Series), 2013. 45(1). 
[15] Madan, R., et al., Energy-efficient cooperative relaying over fading channels with simple relay selection. IEEE Transactions on Wireless Communications, 2008. 7(8): p. 3013-3025.

[16] Chen, Y., et al. Power-aware cooperative relay selection strategies in wireless ad hoc networks. in 2006 IEEE 17th International Symposium on Personal, Indoor and Mobile Radio Communications. 2006. IEEE.

[17] Wei, Y., et al. Energy-Saving Power Allocation Scheme for Relay Networks Based on Graphical Method of Classification. in International Conference on Human Centered Computing. 2016. Springer.

[18] Sun, L., et al., Cooperative communications with relay selection in wireless sensor networks. IEEE Transactions on Consumer Electronics, 2009. 55(2): p. 513-517.

[19] Li, Y., et al., Fair relay selection in decode-and-forward cooperation based on outage priority. Science China Information Sciences, 2013. 56(6): p. 1-10.

[20] Han, L. and J. Mu, Outage Probability of Opportunistic Decode-andForward Relaying over Correlated Shadowed Fading Channels. Wireless Personal Communications, 2016: p. 1-10.

[21] Zhu, Y. and H. Zheng, Understanding the impact of interference on collaborative relays. IEEE Transactions on Mobile Computing, 2008. 7(6): p. 724-736.

[22] Ju, M., K.-S. Hwang, and H.-K. Song, Relay selection of cooperative diversity networks with interference-limited destination. IEEE Transactions on Vehicular Technology, 2013. 62(9): p. 4658-4665.
[23] Shi, C., et al., Distributed interference-aware relay selection for IEEE 802.11 based cooperative networks. IET networks, 2012. 1(2): p. 84-90.

[24] Bletsas, A., et al., A simple cooperative diversity method based on network path selection. IEEE journal on Selected Areas in Communications, 2006. 24(3): p. 659-672.

[25] Fei, L., et al., Relay selection with outdated channel state information in cooperative communication systems. IET Communications, 2013. 7(14): p. 1557-1565.

[26] Bletsas, A., A. Lippnian, and D.P. Reed. A simple distributed method for relay selection in cooperative diversity wireless networks, based on reciprocity and channel measurements. in 2005 IEEE 61st Vehicular Technology Conference. 2005. IEEE.

[27] Wu, Q., X. Zhou, and S. Wang, Relay Selection Considering Successive Packets Transmission in Cooperative Communication Networks. CIT. Journal of Computing and Information Technology, 2014. 22(4): p. 217226.

[28] Brante, G., et al., Distributed fuzzy logic-based relay selection algorithm for cooperative wireless sensor networks. IEEE sensors journal, 2013. 13(11): p. 4375-4386.

[29] Chen, M., T.C.-K. Liu, and X. Dong, Opportunistic multiple relay selection with outdated channel state information. IEEE Transactions on Vehicular Technology, 2012. 61(3): p. 1333-1345.

[30] Mamdani, E.H. and S. Assilian, An experiment in linguistic synthesis with a fuzzy logic controller. International journal of man-machine studies, 1975. 7(1): p. 1-13. 